febrile seizure as an indication for lumbar puncture should be reexamined. Patients who recover consciousness rapidly should be observed, and the tap deferred. (Millichap JJ et al. Methods of investigation and management of infections causing febrile seizures. Presented at AAN 60 ${ }^{\text {th }}$ Annual Meeting, 2008; Pediatr Neurol 2008;39:381-386). In this study, $23 \%$ of febrile seizures were complex, and of these $50 \%$ received LP, a slightly lower percentage than that reported in the Boston study.

\title{
SPEECH AND LANGUAGE DISORDERS
}

\section{FUNCTIONAL MRI NEURAL ACTIVATION PATTERNS IN EARLY AND LATE TALKERS}

Neural activation patterns, produced while listening to and reading words and non-words, were evaluated by functional MRI in 48 school children, mean age 8 years, in a study at Yale School of Medicine, New Haven, CT. Comparison of subsets of early, ontime and late talkers, matched for age, gender and performance IQ, showed that activation in bilateral thalamus and putamen, and left insula and superior temporal gyrus (circuits underlying speech and language) during these tasks was significantly lower in late talkers. Age of language acquisition has effects on reading and language behavior, and on corresponding cortical and subcortical neural circuitry. Late talkers were at risk for reading problems. The findings demonstrate the importance of early language development on formation of critical neural circuits and the need for prompt identification of language delays. (Preston JL, Frost SJ, Mencl WE, et al. Early and late talkers: school-age language, literacy and neurolinguistic differences. Brain August 2010;133:2185-2195). (Respond: Jonathan Preston, Haskins Laboratories, 300 George St, Suite 900, New Haven, CT 06511. E-mail: preston@haskins.yale.edu).

COMMENT. This study underscores previous findings of the effect of age of talking on the development of language and literacy. Talking is strongly related to neural activation patterns, particularly in subcortical regions, putamen and thalamus, regions implicated in the Galaburda studies of dyslexia.

\section{LEFT OCCIPITO-TEMPORAL LOCALIZATION OF READING EPILEPSY}

Researchers at Hopital de la Timone, Marseille, France, report a 28-year-old woman with a history of a febrile seizure at age 4 years and recent onset of partial seizures precipitated by silent reading. Left occipito-temporal localization of the seizures was confirmed by EEG, PET and SPECT. During a seizure, she was unable to understand what she was reading, and after looking up from the page, she saw letters and words, had a feeling of strangeness, right hemi-body jerks, and secondary seizure generalization. Seizures were controlled with carbamazepine. Interictal EEG showed left temporal posterior spikes, and during video-EEG a seizure was recorded $5 \mathrm{~min}$ after silent reading of a woman's magazine, with left occipito-temporal predominance. Interictal PET showed bilateral occipito-temporal hypometabolism with left predominance, and ictal 
SPECT showed hyperperfusion in left occipito-parietal, left lateral temporal, and left inferior frontal regions. Brain MRI was normal. Two varieties of reading epilepsy are differentiated, jaw jerk variant and posterior variant with visual symptoms. (Gavaret M, Guedj E, Koessler L, et al. Reading epilepsy from the dominant temporo-occipital region. J Neurol Neurosurg Psychiatry July 2010;81:710-715). (Dr Martine Gavaret, Service de Neurophysiologie Clinique, Hopital de la Timone, 264 rueSaint Pierre, Marseille 13005, France. E-mail: martine.gavaret@ap-hm.fr).

COMMENT. Bickford RG and colleagues at Mayo Clinic were the first to describe reading epilepsy as a new syndrome (Trans Am Neurol Assoc 1956;81:100102). Seizures were precipitated by reading difficult material, and the cortical or lexical aspects of the seizure provocation were stressed by Bickford and others. In a report from University of Ottawa, Canada, a 24-year-old woman experienced jaw jerking while reading aloud material of medium or high linguistic complexity (Christie $\mathrm{S}$ et al. Epilepsia 1988;29L288-293). These authors found several factors might be involved in seizure precipitation while reading. These included saccadic eye movements, articulation, in addition to difficulty of reading content, and no factor acted solely as the critical stimulus. In a further Mayo Clinic report of 20 patients diagnosed between 1949 and 1989, age at onset ranged from 10 to 46 years (median 17 years). Seizures were myoclonic, involving orofacial and jaw muscles, and generalized tonic-clonic. EEG showed generalized spike or spike and wave discharges in 15 patients and left hemisphere discharges in 5. Seizures responded to valproic acid. (Radhakrishnan K, Silbert PL, Klass DW. Brain 1995;118:75-89).

In an editorial, Koepp MJ of the UK emphasizes that although jaw jerks are the hall mark of reading epilepsy, many other reading-induced ictal symptoms are described: absence seizure, abrupt loss of consciousness, paroxysmal alexia or dyslexia, and prolonged stuttering. Dr Koepp advocates more case studies, similar to that of Gavaret et al, to determine the localization and mechanism of reading epilepsy, in contrast to the current focus on statistical analysis of large random samples in epilepsy research.

\section{SPEECH AND LANGUAGE DYSFUNCTION AND FOCAL EPILEPTIFORM ACTIVITY}

The medical history, EEG recordings, and developmental milestones of 19 children with speech and language dysfunction, and focal epileptiform activity were analyzed for prognostic risk factors in a study at University of Gothenburg, Sweden. Three patterns of language development were observed at follow-up: 1) late start and continued slow development (possible Landau-Kleffner syndrome (LKS) or epileptic language disorder (ELD); 2) late start and deterioration/regression (probable LKS); and 3) normal start and later regression/deterioration (definite LKS). Seizures (complex partial, generalized tonic-clonic, absence, perioral, and eye-lid myoclonic) occurred in 15 patients but were rare. Age at onset was $2-9$ years. Response to AEDs (carbamazepine, etc) was poor, whereas corticosteroids were effective in 8 of 9 cases. Six (32\%) had a family history of seizures. All but one had normal CT or MRI. SPECT showed hypoperfusion in left temporal region. Family history was positive for speech and language delay or reading and writing disability. Early general development was normal 